\title{
Save our sea-level observations
}

\author{
The UK's Permanent Service for Mean Sea Level has operated for 80 years. Such long-term records are \\ invaluable and their diversity must be maintained.
}

Decades of measurements from tide gauges and satellites show that global mean sea level has risen over the past hundred years. A workshop in Liverpool, UK, on 28-29 October 2013 marked the eightieth anniversary of the UK's Permanent Service for Mean Sea Level (http://go.nature.com/dcHBhM) and highlighted the key role of permanency in sea-level observations - and their fragile future in times of limited funding.

We have made impressive progress in our understanding of sea-level rise over the past five years, but significant gaps remain (http://go.nature.com/fwAuB1). Further advances depend on constant and reliable monitoring efforts.

Since the early 1990s, the bulk of information about our expanding seas has come from satellite observations of ocean height and gravity. These data are complemented by a relatively sparse but global network of tide gauges with longerterm measurements, some dating to the late 1800s. Furthermore, since 2000, thousands of floats have been criss-crossing the upper 2,000 m of the world oceans under the Argo programme (http://www.argo.ucsd.edu/). The floats measure temperature and salinity, used to estimate changes in the volume of a given mass of ocean water.

These various observational platforms are built on independent techniques, and they measure different aspects of sea-level rise. They can therefore be used to double-check each other's estimates and to parse out the contributions from increasing mass and volume expansion, as well as disentangling regional and global signals. We need to be able to distinguish between these contributions to project future sea-level rise in a given location.

Despite a sound scientific case, the continuation of these complementary measurements is by no means guaranteed. Replacements for the satellite missions Jason-2, which measures sea surface height, and GRACE, which observes mass changes, are scheduled for launch in 2015 and 2017, respectively - but, at least in the case of GRACE, may not come soon enough to ensure continuous observations. Of course, the 2009 launch failure of NASA's Orbiting Carbon Observatory
(http://go.nature.com/iKeMXh) shows that a planned launch does not necessarily result in an operational satellite. There is thus a case for increasing redundancy and overlap between missions, which also ensures that the resulting records can be knitted together over time.

Even tide gauges, generally considered a continuous stalwart of ocean observations, can be subject to the whims of government funding. Members of the Global Sea Level Observing System (GLOSS), which operates a network of sea-level stations around the globe, are struggling to maintain stations in Africa without further and continuing international support. And despite the hardwon expansion of the network along many tropical coastlines, data from the northern Indian Ocean, as well as most of the Arctic, are still lacking.

Some of these gaps fall in regions that are particularly threatened by rising seas. Efforts at adaptation and defensive measures can only be effective and cost-efficient if we know what to expect. Uninterrupted monitoring is key to quantifying the dangers posed by sea-level rise. It will be money well spent.

\section{The upside of impacts}

\section{The Chelyabinsk fireball highlighted the threat of asteroids and comets. But actually, for life on Earth, impacts may have once played the role of hero.}

Impacts of extraterrestrial bodies are often thought of as life's antagonists in Earth's saga. Ever since the extinction of the dinosaurs was linked to an asteroid impact, scientists have scoured the rock record for evidence of an extraterrestrial cause for other extinction events, and scoured the skies for asteroids on a collision course with our planet. But the effects of impacts are not all deleterious to life: on page 1045, Zita Martins, Mark Price and co-authors demonstrate that impacts could have created life's building blocks. And Kieren Howard and colleagues demonstrate on page 1018 that biological compounds can survive in the melted and ejected products of impact.

Compared with most terrestrial geologic processes, impacts are extreme. When a meteorite or comet hits a planet at speeds of many kilometres per second, it generates a shockwave. As the shockwave propagates away from the point of impact, both target and impactor experience high pressures and temperatures, leading to displacement and ejection of material and formation of a crater within a geological instant. Rocks that are neither vaporized nor melted may be subject to other shock-induced deformation, as is evident within the crystal lattices of minerals where planar features are diagnostic of impact. No other geologic process is quite as, literally, shocking.

But geologic violence can bring creation. As demonstrated on page 1045, when an icy comet hits a rocky planet, the impact could lead to the shock-synthesis of complex amino acids. Similarly, the impact of a meteorite into an ocean could also synthesize complex organic compounds (http://doi.org/fpphvd). On an early Earth with frequent large impacts, shock-synthesis could have generated the key ingredients that life needed to get going.

The seeds of life may also survive the extreme conditions of impact. Once-molten droplets flung from a small crater in Tasmania and quenched to glass were found to contain perfectly preserved organic material from the target ecosystem (page 1018). The find suggests we might examine impact glasses elsewhere for snapshots of life through Earth history and perhaps even life exchanged between planetary bodies.

The same extreme processes that could have helped to create and spread life on Earth continue to threaten it. The Chelyabinsk airburst highlighted the risk presented by even small objects (http://go.nature.com/NblFEP). As we monitor the skies and ponder life's beginnings, impacts continue to bring the drama. 